eher dünn gesät ist, enthält in diesem Band auch die Abteilung „Positionen“ aufschlussreiche Hinweise. Als gesellschaftliches Massenphänomen kann die Bedeutung von Sport als Gegenstand von Anschlusskommunikation realer Sporterlebnisse, von Massenkommunikation und von Anschlusskommunikation über mediale Sport-Events kaum überschätzt werden. Parallelen zwischen Sport- und Medienentwicklung im gesellschaftlichen Modernisierungsprozess scheinen unübersehbar, und viele Aspekte der Mediatisierung von Sport erinnern an ähnliche Beobachtungen zum Verhältnis von Medien und Politik. Symbiotischen Charakter besitzt speziell die (medien-)ökonomische Dimension, wenn Sport als Faktor im Medienmarkt hochrelevant wird (man denke an die öffentliche Diskussion zur Vergabe von Fußball-Übertragungsrechten) und Medien gleichzeitig das Regelwerk ganzer Disziplinen bestimmen.

Zwei empirische Inhaltsanalysen geben interessante Hinweise auf die Medienrepräsentation von konkreten Sportereignissen, deren Ergebnisse (im informatorischen Sinne) zwar vergleichsweise exakt definiert werden können, die in ihrer Interpretation aber beträchtliche Freiheitsgrade aufweisen, weil sich die enthaltenen Bewertungen an den vorherigen Erwartungen orientieren. Neben der Attributionstheorie, die Hans-Jörg Stiehler und Mirko Marr zur Erklärung solcher Phänomene bei der Kommentierung von Turnierauftritten der deutschen Fußball-Nationalmannschaft heranziehen, lässt sich auch die Nachrichtenwertforschung fruchtbar machen: Wiebke Loosens Untersuchung identifiziert mit der Simplizität, dem Nationalbezug, dem Personenbezug und der eben erwähnten Wertigkeit vier durchaus plausible Nachrichtenfaktoren.

Jeder der drei von Gunnar Roters, Walter Klingler und Maria Gerhards herausgegebenen Tagungsbände hinterlässt beim Leser den für Sammelwerke typischen, ambivalenten Eindruck. Die Beiträge sind wissenschaftlich von unterschiedlichem Niveau, unterschiedlicher Relevanz und unterschiedlicher Präzision. Manche Texte erinnern an abgetippte Redemanuskripte, andere dagegen sind voll ausformulierte Papers, die auch eine analytische Dimension jenseits anekdotischer Erfahrungsberichte besitzen. In der Gesamtschau allerdings - und nur so kann man vermutlich dem Konzept „Forum Medienrezeption“ gerecht werden - sum- mieren sich die Einzelwerke zu einem bedeutsamen Literaturkorpus: nämlich zu einem der wenigen Ansätze, das unübersichtliche Feld der Rezeptionsforschung systematisch zu bearbeiten. Betont sei dabei die Transferfunktion, die Tagung, Berichtsband und andere begleitende Materialien erfüllen. Wenn allseits die mangelnde Sichtbarkeit kommunikationswissenschaftlicher Forschung im öffentlichen Diskurs beklagt wird - dem Forum Medienrezeption gelingt zumindest der Dialog zwischen Machern und Beobachtern. Wesentliche Impulse gibt inzwischen die Website (www.medienrezeption.de), deren Mehrwert nicht nur die archivierten Vorträge und entsprechende Druckfassungen ausmacht, sondern die auch die Recherche in einer umfangreichen Literaturdatenbank zum Thema ermöglicht.

Patrick Rössler

\section{Dagmar Hoffmann}

\section{Attraktion und Faszination Medien}

Jugendliche Sozialisation im Kontext von Modernisierung und Individualisierung

Münster: Lit, 2002. - 279 S.

ISBN 3-8258-6042-6

(zugl.: Chemnitz, TU Chemnitz, Diss., 2002)

Diese Dissertation (TU Chemnitz) befasst sich in einer empirischen Vergleichsuntersuchung mit den Medienpräferenzen und mit der Mediennutzung Chemnitzer und Oldenburger Jugendlicher. Der theoretische Bezugsrahmen greift Desiderate der Jugend- und Medienforschung auf. Die Jugendphase wird unter der Perspektive der „Individualisierung“ und der „Modernisierung" diskutiert. Unter der Überschrift „Individualisierung und Instanzen der Sozialisation" setzt sich die Autorin mit den Themen „Familie, Schule und Peergruppe“ auseinander: Die vorliegenden Forschungsergebnisse zum Vergleich von Jugendlichen in Ost und West hätten weniger gravierende Unterschiede zu Tage gefördert, als angenommen wurde. Es fehle hier an „überzeugenden Belegen" für die theoretischen Annahmen. (S. 69) Mit dieser Arbeit will die Autorin eine „soziologische Erklärung “ für die offenen Fragen finden.

Ihre These ist, dass es zwei verschiedene Sozialisationstraditionen in Ost und West gebe. Diese unterschiedlichen Sozialisationskontexte 
werden mit der Unterscheidung von sozialstrukturellen und soziokulturellen Bedingungen erschlossen. Die empirische Untersuchung (Kapitel 5-8) bestand aus einer Befragung von 1011 Jugendlichen im Alter von 10-18 Jahren im Frühjahr 1996. Das durchschnittliche Alter der Befragten betrug 13,8 Jahre. In den Regionen Chemnitz und Oldenburg wurden je 4 Schulklassen befragt: Haupt-, Realschüler und Gymnasiasten in Oldenburg, Mittelschüler und Gymnasiasten in Chemnitz (dort gibt zwei Schultypen). Die Stichprobe umfasste je zur Hälfte Jugendliche aus dem Umland und aus den Städten.

„Die Attraktivität und Faszination der Medien ist für die heutige Jugendgeneration ungebrochen $(. .$.$) Es wird angenommen, dass die ver-$ stärkte Zuwendung Jugendlicher zum Fernsehen und Radio, zu Zeitschriften und Computer Ausdruck einer (post-)modernen Sozialisation ist“. (S. 68) Geprüft werden soll, „ob Jugendliche, die in besonderem Maße den Anforderungen einer individualisierten Gesellschaft gewachsen sind, verstärkt auf Medien zurückgreifen, weil sie diese als Instanz der (Selbst)Sozialisation nutzen" (S. 12; kursiv i. Orig.). Jugendliche in traditionellen, „weniger“ individualisierten Lebensbedingungen seien hier mehr an den allvertrauten Instanzen der Sozialisation interessiert, während die in (post-)modernen Lebenskontexten Medien stärker nutzten.

Um das Problem der „Messbarkeit von Individualisierung " zu lösen und um den Zusammenhang von Sozialisation und Mediennutzung herzustellen, werden für die sozialstrukturellen und soziokulturellen Bedingungen des Aufwachsens folgende Instrumente entwickelt: Für die Individualisierungshypothese sind dieses Instrumente zur „Individualorientierung“, zur „Eltern- und Peerorientierung“, zu "gleichaltrigen Sozialbeziehungen im Jugendalter", zur „regionalen Mobilität" und zu den „Freizeitaktivitäten“ u. a. Die Fragen zu den Medien: Medienbesitz, Programmpräferenzen, Bewertung einzelner Medien, Besonderheiten der Nutzung einzelner Medien (hier insbesondere des Radios) sowie Rezeptions- und Aneignungsstile.

Das überraschende Ergebnis: Es konnten „keine allgemeingültigen Zusammenhänge zwischen der Individualorientierung und der Mediennutzung gefunden werden.“ (S. 229) Besonders die stark „peerorientierten“ Jugendlichen erachteten Medien als sehr wichtig. Ost- deutsche Jugendliche wendeten sich stärker und länger den Medien zu als westdeutsche Jugendliche im gleichen Alter. Medien sind für diejenigen Jugendlichen attraktiv, „die sehr leistungsorientiert sind und sehr an der Entwicklung eines Selbstkonzepts arbeiten." Stärker als die sozialstrukturellen Bedingungen sind nach dieser Untersuchung die soziokulturellen Bedingungen ausschlaggebend für die Mediennutzung (Dauer und Funktion). Überraschend für die Autorin ist ferner der Befund, dass nicht die individualorientierten Jugendlichen sich besonders intensiv mit den Medien beschäftigten, sondern dass es in Ost- und Westdeutschland gleichermaßen die „peerorientierten“ seien. Zwei Erklärungen werden gegeben: Medien seien für diese letztgenannten Jugendlichen Mittel zum Zweck, „den Anforderungen einer sich modernisierenden Gesellschaft zu begegnen“. Medien seien ferner „generalisierte bzw. assoziierte Peers“. Sie hätten eine „sozial-integrative Funktion, sie vermittelten den Jugendlichen das Gefühl, mit anderen in Kontakt zu stehen (hier werden spezifische Rezeptionsstile am Beispiel des Radios dargestellt).

Die Frage ist, ob der hier verwendete Focus auf die „Zuwendung Jugendlicher (individuellbzw. traditional orientierte) zu Medien" ausreichend ist, um Unterschiede und Gemeinsamkeiten in den Lebens- und Mediennutzungsstilen der Jugendlichen in Chemnitz und Oldenburg empirisch zu erschließen. Die These von den zwei verschiedenen Sozialisationstraditionen der befragten Jugendlichen braucht zu ihrer Stützung jedenfalls weitere Untersuchungen. Der Indikator „Mediennutzung und Medienfunktion" kann dieses allein nicht leisten.

Zumal die Medien als Ausdruck der Moderne angeblich eher die Globalisierung vorantreiben als die existierenden regionalen Besonderheiten fördern. Die These, dass die Hinwendung zu Medien gleichzusetzen sei mit Individualisierung und Modernisierung erscheint fraglich. In der Konsequenz werden mit dieser These implizit normative Wertungen vorgenommen: Sind "traditionelle" Jugendliche („Elternorientierte“) eo ipso weniger individualisiert, „unmodern“? Der Eindruck entsteht, es gebe eine lineare Verbindung zwischen den individualisierten „Medienfreaks“, die die Anforderungen der „Moderne“ erfüllen könnten, was eben die „traditionell orientierten“ Ju- 
gendlichen nicht in derselben Weise könnten. Die Mediennutzung als den zentralen Indikator für Individualisierung und Bewältigung der „Moderne“ zu definieren, wird zudem durch die Entwicklung der Medien selbst relativiert: Ihre Ubiquität und ihre fortschreitende Diversifizierung ist mit Prozessen der Normalisierung und Banalisierung verbunden. Medien haben auch eine banale, triviale Seite, sie sind Teil des Alltag der Jugendlichen. Im Übrigen: Medien als solche sind nicht immer und $\mathrm{zu}$ jedem historischen Zeitpunkt ein Medium der „Moderne“ - wie ein Blick z. B. auf die Rolle der Medien nach 1945 in West - und Ostdeutschland zeigt.

Diese Untersuchung leistet eine gründliche Auseinandersetzung mit den theoretischen und empirischen Desideraten der sozialwissenschaftlichen Jugendforschung. Die Ergebnisse der Medienforschung werden diskutiert. Das Vorhaben, eine vergleichende Untersuchung west- und ostdeutscher Jugendlicher unter Berücksichtigung soziokultureller Aspekte anzugehen, ist verdienstvoll, weil es offenbar an dergleichen mangelt. Die Ergebnisse belegen Gemeinsamkeiten aber auch Unterschiede in der Zuwendung und in der Bewertung von Medien zwischen Ost und West. Der Hinweis auf die erklärungsstarken soziokulturellen Bedingungen macht generell den Mangel an Untersuchungen deutlich, die sich mit den kulturellen Differenzen zwischen Regionen, nicht nur denen zwischen Ost und West, beschäftigen. Es ist das Verdienst dieser Untersuchung, dass ihre Fragestellungen und Ergebnisse zugleich ein Ausgangspunkt für eine regional-vergleichende Medienforschung sein könnte.

Ekkehard Sander

\section{Beck'scher TKG-Kommentar}

Hrsg. von Wolfgang Büchner et. al

München: Beck, 2. Aufl., 2000. - 1734 S.

ISBN 3-406-45427-5

Der Beck'sche Kommentar zum Telekommunikationsgesetz bewegt sich mittlerweile selbst wie die Unternehmen in dem von ihm betrachteten Markt in einem hoch kompetativen Umfeld. Es ist dennoch erwartbar, dass er eine starke Marktmacht behält, und das nicht nur, weil er mittlerweile als der etablierteste Kommentar im Telekommunikationsrecht gelten kann. Dazu trägt zum einen die klare, für alle Normen zumindest ähnliche Gliederung der Kommentierung bei. Zum anderen merkt man dem Autorenteam - überwiegend in diesem Bereich ausgewiesene Rechtsanwälte und Justitiare von Telekommunikationsunternehmen - ihre dauerhafte praktische Befassung mit der Materie an. Kaum ein relevantes Problemfeld, das nicht im Kommentar Erwähnung findet; keine Beschränkung auf die Auswertung von Literatur und gefestigter Rechtsprechung, sondern die Berücksichtigung von zahlreichen Entscheidungen der Regulierungsbehörde für Telekommunikation und Post stärkt die Argumentation in den einzelnen Kommentierungen und zeichnet den Kommentar aus.

Die zweite Auflage macht allerdings deutlich, dass ein solcher, von Einzelfällen in der Praxis her synthetisierender Ansatz bei einem dynamischen Feld wie dem Telekommunikationsrecht, das darüber hinaus auch noch durch europarechtliche Einflüsse an Komplexität gewinnt, seinen Preis hat. Es wird zunehmend schwieriger, sich in den zum Teil über eine Seite ziehenden Absätzen die relevanten Informationen zu erschließen. Grundsätze, die die Auslegung leiten und die Darstellung strukturieren können, werden nicht an allen Stellen sichtbar.

Dies ändert allerdings nichts an der Gesamtbewertung: Ohne den Beck'schen TKG-Kommentar kann man schlicht in diesem Rechtsbereich nicht seriös arbeiten. Vielleicht gibt die anstehende Novellierung des TKG Gelegenheit, die vielfältigen, in den Kommentar eingehenden Erfahrungen noch konsequenter zu bündeln und so für den Nutzer noch einfacher erschließbar zu machen.

Wolfgang Schulz

\section{Werner Früh}

\section{Gewaltpotentiale des Fernsehangebots}

Programmangebot und zielgruppenspezifische Interpretation

Wiesbaden: Westdeutscher Verlag, 2001. $221 \mathrm{~S}$.

ISBN 3-531-13706-9

Ausgangspunkt der Studie von Werner Früh ist die wissenschaftliche Debatte um die Wirkung von Gewaltdarstellungen im Fernsehen. Einer der grundlegenden Streitpunkte in dieser De- 